Citation: Hirt C, Claessens SJ, Kuhn M, Featherstone WE (2012) Indirect evaluation of Mars Gravity Model 2011 using a replication experiment on Earth, Studia Geophysica et Geodetica, 56 (2012), 1-xxx, DOI: 10.1007/s11200-011-0468-5

\title{
Indirect evaluation of Mars Gravity Model 2011 using a replication experiment on Earth
}

\author{
C. Hirt, S. J. Claessens, M. Kuhn, W.E. Featherstone \\ Western Australian Centre for Geodesy \& The Institute for Geoscience Research, \\ Curtin University of Technology, GPO Box U1987, Perth, WA 6845, Australia \\ Emails: c.hirt@curtin.edu.au, s.claessens@curtin.edu.au, m.kuhn@curtin.edu.au, \\ w.featherstone@curtin.edu.au
}

\section{Summary}

Curtin University’s Mars Gravity Model 2011 (MGM2011) is a high-resolution composite set of gravity field functionals that uses topography-implied gravity effects at medium- and short-scales ( 125 km to $\sim 3 \mathrm{~km}$ ) to augment the space-collected MRO110B2 gravity model. Ground-truth gravity observations that could be used for direct validation of MGM2011 are not available on Mars's surface. To indirectly evaluate MGM2011 and its modelling principles, an as-close-as-possible replication of the MGM2011 modelling approach was performed on Earth as the planetary body with most detailed gravity field knowledge available. Comparisons among six ground-truth data sets (gravity disturbances, quasigeoid undulations and vertical deflections) and the MGM2011-replication over Europe and North America show unanimously that topography-implied gravity information improves upon space-collected gravity models over areas with rugged terrain. The improvements are $~ 55 \%$ and $\sim 67 \%$ for gravity disturbances, $\sim 12 \%$ and $\sim 47 \%$ for quasigeoid undulations, and $\sim 30 \%$ to $\sim 50 \%$ for vertical deflections. Given that the correlation between space-collected gravity and topography is higher for Mars than Earth at spatial scales of a few $100 \mathrm{~km}$, topographyimplied gravity effects are more dominant on Mars. It is therefore reasonable to infer that the MGM2011 modelling approach is suitable, offering an improvement over space-collected Martian gravity field models. 
Keywords: Mars gravity field, Earth gravity field, gravity, topography, forward-modelling

\section{Introduction}

Recently, Curtin University’s Western Australian Centre for Geodesy has released Mars Gravity Model 2011 (MGM2011), a high-resolution model of Mars’s gravity field (http://geodesy.curtin.edu.au/research/models/mgm2011). MGM2011 (Hirt et al., 2012a) is a composite model constructed and represented as grids of various gravity field functionals in the spatial domain based on satellite-implied gravity (SIG) via the MRO110B2 model (Konopliv et al., 2011) and topography-implied gravity (TIG) based on elevation data from high-resolution Mars laser altimetry (Smith et al., 2001). It provides grids of estimates of surface gravity accelerations, disturbances and surface vertical deflections down to scales of $\sim 3 \mathrm{~km}$ and quasigeoid undulations at scales of $\sim 3 \mathrm{~km}$ to $\sim 125 \mathrm{~km}$. The innovation of MGM2011 lies in the use of TIG information on Mars to augment SIG at medium and shortscales ( 125 km to $\sim 3 \mathrm{~km})$.

MGM2011 was developed based on tried and tested methodologies from Earth gravity field modelling (e.g., Forsberg, 1984; Pavlis et al., 2007; Hirt, 2010; Hirt et al., 2010a,b); see Section 2. Because there are no ground-truth gravity-related observations available on Mars's surface for a direct model evaluation, we evaluate the MGM2011 gravity modelling techniques from an as-close-as-possible replication on Earth, thus it is an indirect and implict evaluation. The replication uses exactly the same modelling approach with similar parameters (Section 2). The Earth's short-scale gravity field is dominated by the gravitational attraction of the topography (e.g., ibid.; Torge, 2001). Because both planets possess significant topographic masses, and variations in elevation are even larger on Mars than on Earth, we believe that our replication experiment not only indirectly validates the MGM2011 modelling technique, but also provides indirect insight into the expected performance of MGM2011.

From the abundance of existing ground-truth gravity field data sets on Earth, we use observed gravity acelerations, Helmert (surface) vertical deflections and quasigeoid undulations in Europe, the United States and Canada (Section 3) to test the MGM2011 modelling principles and products, and to benchmark the (expected) improvements conferred 
by our topography-based forward-modelling of short-scale gravity effects on Mars (Section 4). The data sets were chosen such that all of the MGM2011 functionals (gravity disturbances as radial derivatives of the disturbing potential, vertical deflections as horizontal derivatives, and quasigeoid undulations being a linear functional of the disturbing potential) are evaluated indirectly. We present results of cross-comparisons between TIG and SIG on Earth and Mars (Section 5), demonstrating that the topography is not only a major contributor to Mars's gravity field, but also more dominant for Mars than Earth at medium and short scales. Finally, the results of our MGM2011 replication experiment are discussed, and inferences for MGM2011 are made in Section 6.

On Mars, topography-based gravity field modelling is currently - to the best of our knowledge - the only solution to derive information on the (expected) short-wavelength gravity field that cannot be sensed by satellite tracking. On Earth, direct observations of the gravity field are routinely used to model the gravity field down to km-scales (e.g., Torge, 2001). It is acknowledged that the abundance of direct observations carry much more complete information on anomalous gravity field features, as TIG estimates rely on constant mass-density and other assumptions. The scope of the present study is not to model the Earth's gravity field as accurately as possible from terrestrial gravity field observations, but to test the MGM2011 modelling approach in an 'Earth laboratory'. Therefore, we deliberately use TIG functionals at spatial scales shorter than $\sim 125 \mathrm{~km}$ to augment SIG from recent satellite gravity field missions (Pail et al., 2010), as a terrestrial as-close-as-possible replication of MGM2011.

\section{The MGM2011 replication experiment}

MGM2011 constituents are (1) a normal or reference gravity field evaluated on the Martian topography, (2) a gravitational potential model (SIG), and (3) TIG effects from Newtonian forward modelling. The normal gravity field approximates Mars as rotating constant massdensity ellipsoid; the SIG delivers observed anomalies of Mars's gravity field (with respect to the mass-ellipsoid) down to scales of $\sim 125 \mathrm{~km}$ (spherical harmonic degree 85 ), and TIG effects serve as augmentation of the (expected) high-frequency gravity field functionals at scales from $\sim 125$ down to $\sim 3 \mathrm{~km}$, that are not or insufficiently resolved by the SIG. 
Next, the modelling of the three constituents is explained for Mars (MGM2011 original model) and Earth (MGM2011 replication) in a comparitive manner, see also Table 1.

Table 1 Comparison of data, parameters and models used to create Mars Gravity Model 2011 (MGM2011) and the MGM2011 replication on Earth

\begin{tabular}{|c|c|c|c|}
\hline Category & Parameter/ Data set & $\begin{array}{l}\text { Mars (MGM2011) } \\
\text { (Hirt et al., 2012a) }\end{array}$ & $\begin{array}{l}\text { Earth (replication) } \\
\text { this study }\end{array}$ \\
\hline \multirow[t]{2}{*}{ Geometry of planet } & Mean radius & $\sim 3389 \mathrm{~km}$ & $\sim 6371 \mathrm{~km}$ \\
\hline & Scale of 1 degree & $\sim 59.2 \mathrm{~km}$ & $\sim 110 \mathrm{~km}$ \\
\hline \multirow[t]{2}{*}{ Geodetic Reference System } & Name & $\begin{array}{l}\text { MGRS } \\
\text { (Hirt et al., 2012a) }\end{array}$ & $\begin{array}{l}\text { GRS80 } \\
\text { (Moritz 2000) }\end{array}$ \\
\hline & $\begin{array}{l}\text { Defining parameters } \\
\text { a,b, GM, omega }\end{array}$ & $\begin{array}{l}3395428 \mathrm{~m} \\
3377678 \mathrm{~m} \\
4.2828372 \times 10^{13} \mathrm{~m}^{3} \mathrm{~s}^{-2} \\
7.0882181 \times 10^{-05} \mathrm{rad} \mathrm{s}^{-1}\end{array}$ & $\begin{array}{l}6378137 \mathrm{~m} \\
6356752.3141 \mathrm{~m} \\
398600.5 \times 10^{9} \mathrm{~m}^{3} \mathrm{~s}^{-2} \\
7.292115 \times 10^{-05} \mathrm{rad} \mathrm{s}^{-1}\end{array}$ \\
\hline \multirow[t]{2}{*}{ Space-collected gravity model } & Name of model & $\begin{array}{l}\text { MRO110B2 } \\
\text { (Konopliv et al., 2011) }\end{array}$ & $\begin{array}{l}\text { GOCO01S } \\
\text { (Pail et al., 2010) }\end{array}$ \\
\hline & $\begin{array}{l}\text { Harmonic degree/ } \\
\text { Spatial scale }\end{array}$ & $\begin{array}{l}\text { up to } n=85 \\
(125 \mathrm{~km})\end{array}$ & $\begin{array}{l}\text { up to } n=160 \\
(124 \mathrm{~km})\end{array}$ \\
\hline \multirow[t]{2}{*}{ Detailed elevation data } & Name of model & $\begin{array}{l}\text { MOLA } \\
\text { (Smith et al., 2001) }\end{array}$ & $\begin{array}{l}\text { SRTM V4.1 } \\
\text { (Jarvis et al., 2008), } \\
\sim 1 \mathrm{~km} \text { release }\end{array}$ \\
\hline & $\begin{array}{l}\text { Spatial resolution at } \\
\text { the equator }\end{array}$ & $1 / 64^{\circ} \equiv 0.93 \mathrm{~km}$ & $1 / 120^{\circ} \equiv 0.92 \mathrm{~km}$ \\
\hline \multirow[t]{2}{*}{$\begin{array}{l}\text { Long-wavelength } \\
\text { elevation data }\end{array}$} & Name of model & $\begin{array}{l}\text { MarsTopo719 } \\
\text { (Wieczorek ,2007) }\end{array}$ & $\begin{array}{l}\text { DTM2006 } \\
\text { (Pavlis et al., 2007) }\end{array}$ \\
\hline & Harmonic degrees & 0 to 85 & 0 to 160 \\
\hline \multirow[t]{5}{*}{ Forward-modelling } & $\begin{array}{l}\text { Construction of } \\
\text { RTM data }\end{array}$ & $\begin{array}{l}\text { MOLA minus } \\
\text { MarsTopo719 }\end{array}$ & $\begin{array}{l}\text { SRTM minus } \\
\text { DTM2006.0 }\end{array}$ \\
\hline & $\begin{array}{l}\text { Spatial scales taken } \\
\text { from topography }\end{array}$ & $125 \mathrm{~km}$ to $0.93 \mathrm{~km}$ & $124 \mathrm{~km}$ to $0.92 \mathrm{~km}$ \\
\hline & grid resolution & $3^{\prime} \equiv 2.96 \mathrm{~km}$ & $1.5^{\prime} \equiv 2.75 \mathrm{~km}$ \\
\hline & integration radius & $400 \mathrm{~km}$ & $400 \mathrm{~km}$ \\
\hline & Software & TC_dg_Mars.f & TC_dg.f \\
\hline \multirow[t]{3}{*}{ Ground-truth data } & Gravity & Not available & Switzerland, Canada \\
\hline & $\begin{array}{l}\text { Deflections of the } \\
\text { vertical }\end{array}$ & Not available & Europe, US \\
\hline & Geoid undulations & Not available & Germany, US \\
\hline
\end{tabular}




\subsection{Normal gravity/ Geodetic Reference System}

On Earth, normal gravity is often modelled based on the parameters of the Geodetic Reference System 1980 (GRS80, Moritz, 1980). Four parameters (ellipsoidal semi-major axis, semi-minor axis [or alternatively the dynamic form factor], product of the planetary mass and universal gravitational constant, and rotation rate) are used to fully define the geometry, gravitational attraction, and acceleration due to rotation of a reference ellipsoid. In analogy to Moritz (1980), we have replicated the GRS80 concept for Mars, yielding the Mars Geodetic Reference System MGRS. The defining parameters of GRS80 and MGRS are given in Table 1, see also Ardalan et al., (2009) and Hirt et al., (2012a).

Both on Earth and Mars, a geodetic reference system is used to compute normal gravity accelerations at the surface of the topography using the formula of SomiglianaPizzetti (e.g., Torge 2001, p.106) and a second-order Taylor expansion to describe the attenuation of gravity with height (e.g., Featherstone 1995, Torge, 2001, p 110). For both planets, the zonal harmonic coefficients implied by the respective geodetic reference system are subtracted from the SIG model coefficients to account for each planet's oblate ellipticity (cf. Smith 1998).

\subsection{Satellite-implied gravity (SIG)}

Both for Earth and Mars, we use SIG to model the long-wavelength gravity field, down to scales of $125 \mathrm{~km}$. MGM2011 uses MRO110B2 (Konopliv et al., 2011) to harmonic degree 85; the Earth-based MGM2011 replication makes use of GOCO01S (Pail et al., 2010) to harmonic degree 160. Most importantly, the harmonic degrees chosen translate into nearly identical spatial scales of $\sim 125 \mathrm{~km}$ for Earth and Mars (Table 1).

MRO110B2 relies on tracking-data to the Mars Global Surveyor, Odyssey and Mars Reconnaissance Orbiter spacecraft (Konopliv et al., 2011). GOCO01S (Pail et al., 2010) is a combined solution of GRACE (Gravity Recovery and Climate Change Experiment) satelliteto-satellite tracking data (e.g., Tapley et al., 2004) and GOCE (Gravity Field and Steady-state Ocean Circulation Explorer) satellite gradiometry (e.g., Rummel et al., 2011).

Both on Earth (GOCO01S) and Mars (MRO110B2), we use the potential model coefficients to derive gravity disturbances, quasigeoid undulations (aka height anomalies) and vertical deflections through spherical harmonic synthesis. To account for the effect of gravity 
attenuation with height, the potential models are evaluated at the topographic surface, as represented through the SRTM (Shuttle Radar Topography Mission) elevation model (Jarvis et al., 2008) on Earth and MOLA (Mars Orbiter Laser Altimeter) data (Smith et al., 2001) on Mars.

\subsection{Topography-implied gravity (TIG)}

The short-scale gravity field - that is largely omitted by the potential models - is sourced from high-pass-filtered topography, which is also known as a residual terrain model (RTM, Forsberg, 1984). The well-established technique of Newtonian forward-modelling (e.g., Nagy et al., 2000; Kuhn and Featherstone, 2003; Pavlis et al., 2007; Hirt, 2010) is used to compute the gravitational effects (gravity disturbances, quasigeoid undulations, vertical deflections), as implied by the RTM topography. We construct the RTM topography as difference of detailed elevation data (with km-resolution) and a long-wavelength sphericalharmonic topography expanded to the same harmonic degree used to synthesise the SIG component of the model.

For the detailed elevation data, we use the $\sim 1 \mathrm{~km}\left(1 / 64^{\circ}\right)$ MOLA topography (Smith et al., 2001) for Mars and the $\sim 1 \mathrm{~km}\left(1 / 120^{\circ}\right)$ SRTM topography (Jarvis et al., 2008) for Earth. The spatial detail provided by both products is thus comparable. As long-wavelength topography, we use MarsTopo719 (Wieczorek, 2007) to harmonic degree 85 and DTM2006.0 (Pavlis et al., 2007) to degree 160 on Earth. Again, for both planets these harmonic degrees translate into spatial scales of $\sim 125 \mathrm{~km}$.

The resulting RTM data sets were converted to TIG functionals (gravity, quasigeoid, vertical deflections) using the TC software (Forsberg, 1984) for Earth (tc_dg.f) and a TCvariant (tc_dg_mars.f) for Mars. In both cases, we computed gravity effects at dense grids of $\sim 3 \mathrm{~km}$ resolution (that is, 3'x3' for Mars and 1.5'x1.5' for Earth). The integration radius was set to $400 \mathrm{~km}$ for MGM2011 and its replication on Earth, which is more than sufficient given the oscillating character of the RTM (the longest wavelengths contained in the RTM are 125 $\mathrm{km})$, and gravity attenuation with increasing distance.

The Newtonian forward-modelling is based on a constant mass-density assumption for the residual topography on each planet. We use standard rock mass-densities of 2670 $\mathrm{kg} / \mathrm{m}^{3}$ for Earth (e.g., Torge, 2001) and $2900 \mathrm{~kg} / \mathrm{m}^{3}$, a mean mass-density value for Mars 
(Wieczorek and Zuber, 2004). Therefore, the TIG from RTM-data only ever approximates the true short-scale gravity effects of Earth and Mars to some extent. Mass-density anomalies of the real topography (with respect to the constant mass-density) are not modelled. Likewise, any short-scale anomalies present in the interior of Earth and Mars remain unresolved. The Newtonian forward-modelling also relies on the assumption of no isostatic compensation. Nonetheless, inclusion of TIG yields a more complete and precise description of the gravity field than a satellite-only model, as will be seen from our ground-truth comparisons (Section 3).
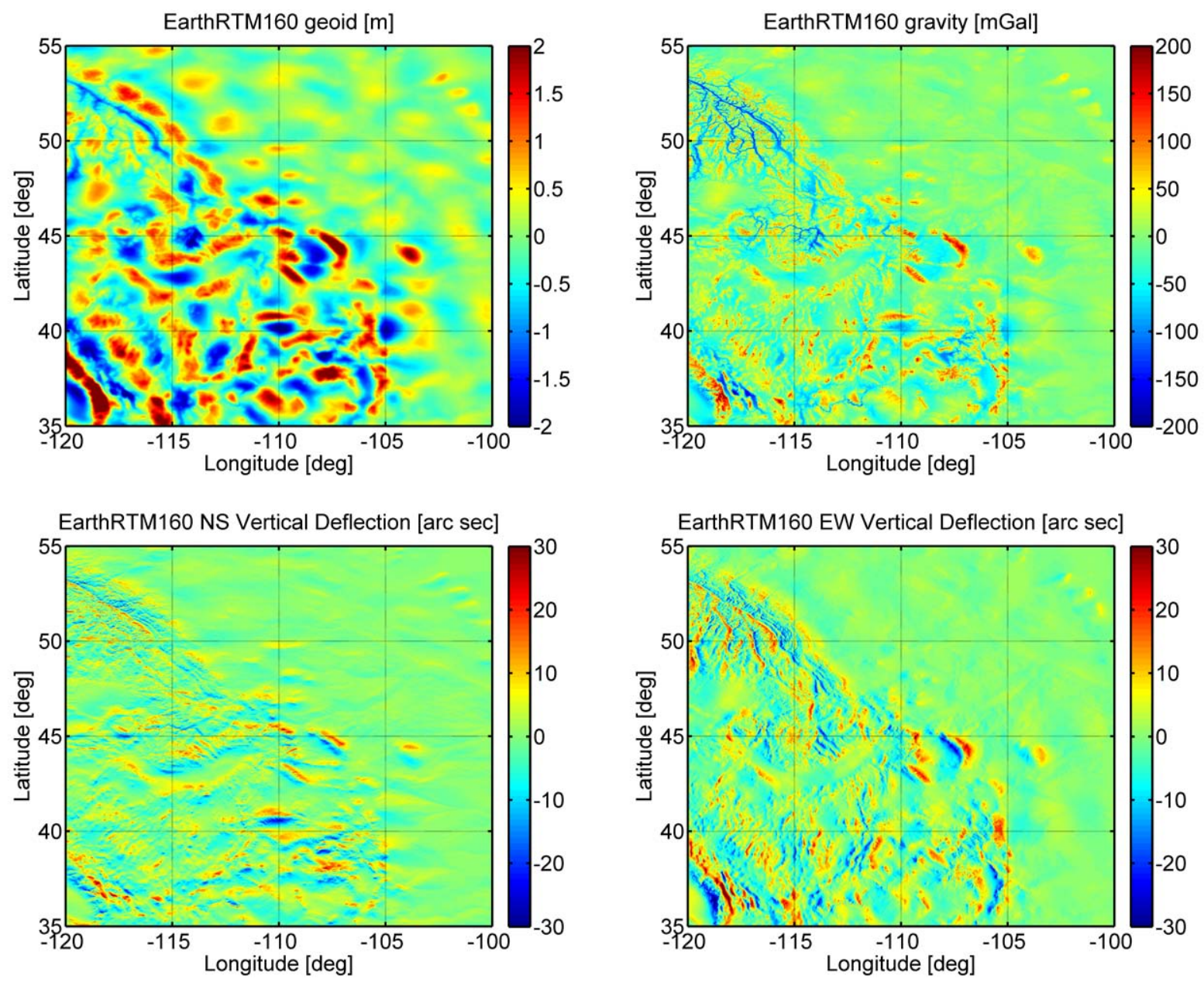

Fig. 1. Earth TIG functionals at spatial scales of $124 \mathrm{~km}$ to $0.92 \mathrm{~km}$. Top left: RTM quasigeoid, top right: RTM gravity, bottom left: RTM NS vertical deflection, bottom right: RTM EW vertical deflection. Computation area covers parts of the United States and Canada. 


\subsection{Computations on Mars and Earth}

For MGM2011, the methodology outlined in Sections 2.1 to 2.3 was applied over a 3' grid covering the entire surface of Mars. On Earth, the MGM2011 modelling was replicated at 1.5' resolution with the parameters reported in Table 1 over two test areas on Earth with rugged topography and where ground-truth data is available:

(1) Central Europe $\left(4^{\circ}<\right.$ longitude $\lambda<16^{\circ} ; 45^{\circ}<$ latitude $\left.\varphi<56^{\circ}\right)$,

(2) North America $\left(240^{\circ}<\lambda<260^{\circ} ; 35^{\circ}<\varphi<55^{\circ}\right)$.

Figure 1 exemplifies the TIG functionals over the North American Rocky Mountains. The spatial scales contained in the TIG functionals are $124 \mathrm{~km}$ to $0.92 \mathrm{~km}$ (Table 1), which is commensurate with the TIG component of MGM2011. Both on Earth and Mars, the TIG functionals are simply added to the SIG in order to improve upon the satellite-only model of each planet's gravity field. Normal gravity (as implied by the GRSs) is added to the TIG and SIG to yield gravity accelerations at the physical surface.

\section{Terrestrial ground-truth data sets}

Table 2 summarises the six ground-truth data sets that we use to benchmark the MGM2011 replication on Earth. We use direct observations of gravity functionals as ground truth: Swiss and Canadian gravity disturbances, European and U.S. Helmert vertical deflections, German quasigeoid undulations and U.S geoid undulations that have been converted to quasigeoid undulations. Though the test areas chosen represent all types of topography (flat, medium elevated and rugged), the selection of our data sets places some emphasis on rugged terrain (European Alps, U.S./Canadian Rocky Mountains), as is often found over Mars’s southern hemisphere (cf. Section 5).

Table 2 Ground-truth data sets used to test the performance of the MGM2011 approach

\begin{tabular}{|l|l|l|l|}
\hline $\begin{array}{l}\text { Observed } \\
\text { Functional }\end{array}$ & $\begin{array}{l}\text { Area name } \\
\text { boundaries } \\
\text { number of stations }\end{array}$ & $\begin{array}{l}\text { Method used to } \\
\text { derive the gravity } \\
\text { functional }\end{array}$ & $\begin{array}{l}\text { Data set name and provider/ } \\
\text { Citation }\end{array}$ \\
\hline Gravity & $\begin{array}{l}\text { Switzerland } \\
5.5^{\circ}<\lambda<11^{\circ} ; 45.5^{\circ}<\varphi<58^{\circ} \\
31598\end{array}$ & $\begin{array}{l}\text { Terrestrial } \\
\text { Gravimetry }\end{array}$ & $\begin{array}{l}\text { Swiss National Gravity set } \\
\text { Swiss Geodetic Commission \& } \\
\text { Swisstopo, (Marti, 2004) }\end{array}$ \\
\hline Gravity & Canada (Alberta) & Terrestrial & Canadian Gravity Database \\
\hline
\end{tabular}




\begin{tabular}{|c|c|c|c|}
\hline & $\begin{array}{l}240^{\circ}<\lambda<250^{\circ} ; 49^{\circ}<\varphi<55^{\circ} \\
10330\end{array}$ & Gravimetry & $\begin{array}{l}\text { Natural Resources Canada } \\
(\text { NRC 2011) }\end{array}$ \\
\hline $\begin{array}{l}\text { Vertical } \\
\text { deflections } \\
(\mathrm{DoV})\end{array}$ & $\begin{array}{l}\text { Europe (Switzerland, } \\
\text { Germany, Netherlands) } \\
6^{\circ}<\lambda<11.5^{\circ} ; 45^{\circ}<\varphi<56^{\circ} \\
1011\end{array}$ & $\begin{array}{l}\text { Astronomical } \\
\text { observations }\end{array}$ & $\begin{array}{l}\text { European Vertical deflections } \\
\text { Swiss Geodetic Commission \& } \\
\text { ETH Zurich \& U Hannover } \\
\text { (Hirt et al. 2010b) }\end{array}$ \\
\hline $\begin{array}{l}\text { Vertical } \\
\text { deflections } \\
(\mathrm{DoV})\end{array}$ & $\begin{array}{l}\text { Western United States } \\
240^{\circ}<\lambda<248^{\circ} ; 40^{\circ}<\varphi<50^{\circ} \\
7977\end{array}$ & $\begin{array}{l}\text { Gradients of } \\
\text { USGG2009 at } \\
\text { scattered points }\end{array}$ & $\begin{array}{l}\text { USDOV2009 } \\
\text { National Geodetic Survey } \\
(\text { NGS 2011a) }\end{array}$ \\
\hline $\begin{array}{l}\text { GPS/levelling } \\
\text { (quasi)geoid } \\
\text { undulations }\end{array}$ & $\begin{array}{l}\text { Germany } \\
5.5^{\circ}<\lambda<16^{\circ} ; 47^{\circ}<\varphi<56^{\circ} \\
675\end{array}$ & $\begin{array}{l}\text { GPS-heighting and } \\
\text { spirit levelling }\end{array}$ & $\begin{array}{l}\text { German Bundesamt für } \\
\text { Kartographie und Geodäsie (BKG) } \\
\text { (Ihde and Sacher, 2002) }\end{array}$ \\
\hline $\begin{array}{l}\text { GPS/levelling } \\
\text { geoid } \\
\text { undulations }\end{array}$ & $\begin{array}{l}\text { Western United States } \\
240^{\circ}<\lambda<260^{\circ} ; 35^{\circ}<\varphi<50^{\circ} \\
1647\end{array}$ & $\begin{array}{l}\text { GPS-heighting and } \\
\text { spirit levelling }\end{array}$ & $\begin{array}{l}\text { GPSBM2009 } \\
\text { National Geodetic Survey } \\
(N G S 2011 b)\end{array}$ \\
\hline
\end{tabular}

- We use the national gravity data set of Switzerland (31,598 stations, Marti, 2004) and a selection of the Canadian gravity data over the Alberta region $\left(10^{\circ} \times 6^{\circ}, 10,330\right.$ stations, $N R C$, 2011). Both data sets are the result of observed terrestrial gravimetry. The Swiss gravity data is accurate at the $0.1 \mathrm{mGal}$ level or better (U. Marti, pers. comm. 2010) and the Canadian gravity about $0.3 \mathrm{mGal}$ (M. Véronneau, pers. comm. 2011), which is well below the expected MGM2011 replication accuracy (cf. Table $3)$.

- Over Europe, we use a set of 1,000 vertical deflections (DoV) that originates from astronomical observations. The data are concentrated over Switzerland and the German Alps, with some parts covering Northern Germany and the Netherlands. The DoV accuracy is at the $0.5^{\prime \prime}$ level or better ( Hirt et al., 2010b). Over a $8^{\circ} \times 10^{\circ}$ area located in the U.S., we use a set of 7,977 gravimetric DoVs, derived at randomly scattered locations as horizontal gradients of the USGG2009 gravimetric geoid model (Wang et al., 2011). The U.S. DoVs are not independent from the RTM-quantities because topographic information was also used in USGG2009. Nonetheless, we consider this data set to have sufficient ground-truth quality because USGG2009 gravimetric DoVs were found to be in $\sim 1$ " agreement with independent astronomical DoVs (Wang et al., 2011) and the SIG/TIG DoVs are no more accurate than a few arc 
seconds (see Table 4). The U.S. astronomical DoVs (e.g., Jekeli, 1999) were not available to us.

- Directly observed quasigeoid undulations are available at GPS/levelling points in Germany (676 stations) and geoid undulations in the U.S. part of our test area $\left(20^{\circ} \times 15^{\circ}\right)$. The German GPS/levelling points are accurate to few $\mathrm{cm}$ (Ihde and Sacher, 2002), and the U.S. data is somewhat below this level (discrepancies of the 3$9 \mathrm{~cm}$ with respect to the USGG2009 geoid are reported by Wang et al., 2011). To be compatible with quasigeoid undulations from SIG (GOCO01S), a conversion from geoid to quasigeoid undulations was performed for the U.S.; see Section 4.3.

\section{Evaluation results}

In all comparisons, we bicubically interpolated the $1.5^{\prime}$ grids of the SIG and TIG functionals to the locations of the ground-truth stations, assming all coordinates to be geocentric. Here and in the remainder of this evaluation, we always compare ground-truth observations with (1) the SIG functionals, and (2) the sum of SIG and TIG functionals. The latter case is the replication of the MGM2011 approach on Earth. The former case represents the use of information from the SIG only, thus omits the short-scale gravity field. In both cases, the SIG functionals are from GOCO01S, synthesised in a spectral band of harmonic degrees 2 to 160.

\subsection{Results using ground-truth gravity disturbances}

For the Swiss and Canadian test areas, Table 3 reports the descriptive statistics of the differences observed minus SIG gravity disturbances as well as of the differences observed minus (SIG+TIG) gravity disturbances. Observed gravity disturbances are the difference between observed gravity and normal gravity at the gravity station. In each case, a regional gravimetric quasi/geoid model has been used to determine the ellipsoidal heights of the gravity observations, thus permitting the computation of gravity disturbances.

From Table 3, TIG-augmentation of SIG reduces the residuals by $\sim 55 \%$ (Switzerland) and $\sim 67 \%$ (Canada), to the level of a few 10s of mGal RMS. This improvement is also seen in Fig. 2 (Switzerland) and Fig. 3 (Canada). TIG improves the agreement in mountain regions often by $\sim 100 \mathrm{mGal}$ to $200 \mathrm{mGal}$, yielding a much improved agreement with the ground-truth observations. This is seen over the entire Swiss Alps (Fig. 
2) as well as over the Canadian Rocky Mountains (Fig. 3). This behaviour demonstrates that at km-scales in rugged terrain, the Earth's gravity field is often dominated by the topographic masses.

Table 3 Comparisons using ground-truth gravity disturbances

\begin{tabular}{|c|c|c|c|c|c|c|c|}
\hline \multirow[t]{2}{*}{ Dataset } & \multirow[t]{2}{*}{ \#Pts } & \multirow[t]{2}{*}{ Difference } & \multicolumn{4}{|c|}{ Descriptive Statistics [mGal] } & \multirow{2}{*}{$\begin{array}{c}\text { Improve } \\
\text { ment }\end{array}$} \\
\hline & & & Min & Max & Mean & RMS & \\
\hline $\begin{array}{l}\text { Swiss } \\
\text { gravity }\end{array}$ & 31,598 & $\begin{array}{l}\text { Observed-SIG } \\
\text { Observed-(SIG+TIG) }\end{array}$ & $\begin{array}{l}-207 \\
-168\end{array}$ & $\begin{array}{l}166 \\
167\end{array}$ & $\begin{array}{c}-44 \\
-4\end{array}$ & $\begin{array}{l}66 \\
30\end{array}$ & $55 \%$ \\
\hline $\begin{array}{l}\text { Canadian } \\
\text { gravity }\end{array}$ & 10,330 & $\begin{array}{l}\text { Observed-SIG } \\
\text { Observed-(SIG+TIG) }\end{array}$ & $\begin{array}{l}-185 \\
-147\end{array}$ & $\begin{array}{l}118 \\
81\end{array}$ & $\begin{array}{c}-10 \\
-1\end{array}$ & $\begin{array}{l}41 \\
14\end{array}$ & $67 \%$ \\
\hline
\end{tabular}
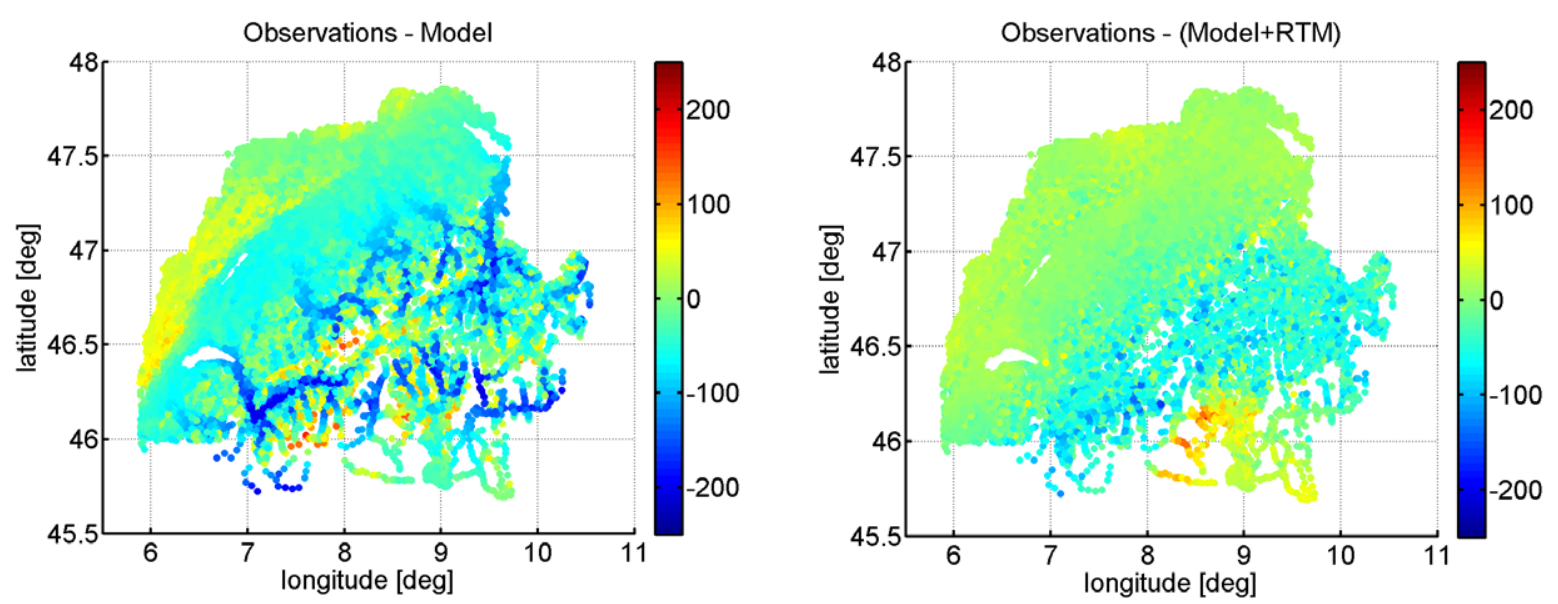

Fig. 2. Gravity comparisons over Switzerland. Left: ground-truth gravity minus GOCO01S (band 2 to 160) gravity. Right: ground-truth gravity minus GOCO01S gravity minus RTM-modelled gravity. GRS80 normal gravity at station height subtracted from observed gravity. Unit in mGal.

Figure 2 exemplifies the limitations of the TIG-augmentation approach. It only ever delivers the gravitational effects of the visible topographic masses based on the assumption of a constant mass-density and isostatically uncompensated topography. Local mass-density anomalies in the topography, intra-crustal mass-density anomalies and isostatic compensation (over the entire Swiss Central Alps) remain unmodelled, so show up in the residuals (Fig. 2 right). The Ivrea body, a mass-density surplus of near-surface intra-crustal material centred at $46^{\circ} \mathrm{N}, 8.5^{\circ} \mathrm{E}$ (Bürki, 1989), is not modelled by the RTM, as seen by the positive residuals in Fig. 2. In case of the Swiss Central Alps, the RTM-modelling does not take into account 
isostatic compensation at scales shorter $\sim 125 \mathrm{~km}$, so overestimates the signals generated by the real (isostatically compensated) topography. This is seen by the negative footprint over the Swiss Alps.

Modelling these isostatic and density-contrast effects obviously requires observations, which exist on Earth (e.g., the Swiss national gravity set) while not [yet] available on Mars. Hence, at scales shorter than $125 \mathrm{~km}$, MGM2011 neglects all features that do not originate from "constant mass-density topography". Conversely, the TIG component of the MGM2011 replication delivers large parts of high-frequency gravity signals, as generated by the visible topography, and this yields much improved gravity estimates in very rugged terrain (Table 3, Fig. 2, 3).
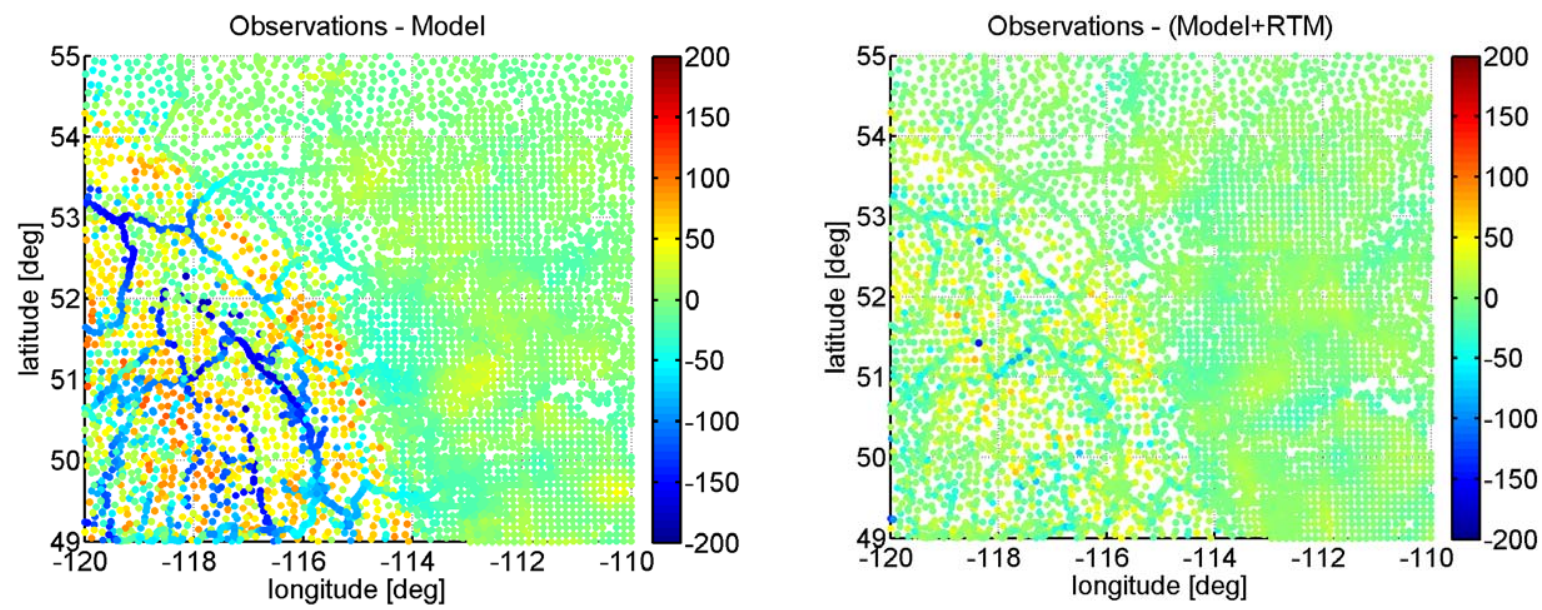

Fig. 3. Gravity comparisons over Canada (Alberta). Left: ground-truth gravity minus GOCO01S (band 2 to 160) gravity. Right: ground-truth gravity minus GOCO01S gravity minus RTM gravity. GRS80 normal gravity at station height subtracted from observed gravity. Unit in mGal.

\subsection{Results using ground-truth vertical deflections}

From Table 4, TIG augmentation reduces the residuals between observed and GOCO01S vertical deflections by $>30 \%$ for Europe and by $>40 \%$ over the Rocky Mountains. Given that deflections possess significant spectral energy at shorter scales (e.g., Jekeli 1999), TIG considerably reduces the variability of the residuals in mountainous regions. 
Table 4 Comparisons using ground-truth vertical deflections, unit is arc seconds

\begin{tabular}{|c|c|c|c|c|c|c|c|}
\hline \multirow[t]{2}{*}{ Dataset } & \multirow[t]{2}{*}{ Points } & \multirow[t]{2}{*}{ Difference } & \multicolumn{4}{|c|}{ Descriptive Statistics [sec] } & \multirow{2}{*}{$\begin{array}{c}\text { Improve } \\
\text { ment }\end{array}$} \\
\hline & & & Min & Max & Mean & RMS & \\
\hline European & \multirow[t]{2}{*}{1011} & Observed-SIG & -27 & 29 & 1 & 7.3 & \multirow{2}{*}{$31 \%$} \\
\hline NS-DoV & & Observed-(SIG+TIG) & -14 & 24 & 0 & 5.0 & \\
\hline European & \multirow[t]{2}{*}{1011} & Observed-SIG & -24 & 26 & 1 & 6.2 & \multirow{2}{*}{$33 \%$} \\
\hline EW-DoV & & Observed-(SIG+TIG) & -24 & 14 & 0 & 4.1 & \\
\hline US & \multirow[t]{2}{*}{7977} & Derived-SIG & -17 & 16 & 0 & 3.8 & \multirow{2}{*}{$41 \%$} \\
\hline NS-DoV & & Derived -(SIG+TIG) & -11 & 10 & 0 & 2.3 & \\
\hline US & \multirow[t]{2}{*}{7977} & Derived -SIG & -28 & 27 & 0 & 5.1 & \multirow{2}{*}{$48 \%$} \\
\hline EW-DoV & & Derived -(SIG+TIG) & -11 & 14 & 0 & 2.7 & \\
\hline
\end{tabular}

NS = North-South, EW= East-West

\subsection{Results using ground-truth quasigeoid undulations}

In order to be compatible with GOCO01S quasigeoid undulations, we converted the U.S. GPS/levelling geoid undulations (Wang et al., 2011) to quasigeoid undulations using Rapp’s approach (Rapp, 1997). We computed the C2-term (Rapp, 1997, p.283) using U.S. Bouguer gravity anomalies (NGDC, 2011) and the Helmert orthometric station heights. Because GOCO01S was synthesised at the height of the topography and not at the ellipsoid (Section 2.2), Rapp’s C1-term (ibid) is implicitly accounted for. For the German GPS/levelling quasigeoid undulations (Ihde and Sacher, 2002), geoid-to-quasigeoid conversion is not required.

Table 5 reports the comparison involving observed quasigeoid undulations in Germany (Fig. 4) and derived quasigeoid undulations in the U.S. (Fig. 5). To account for vertical datum offsets, a bias fit was applied over Germany and a bias-tilt-fit over the U.S (the U.S. GPS/levelling data is subjected to long-wavelength errors of the vertical datum, cf. Wang et al., 2011). Adding TIG to the GOCO01S quasigeoid undulations reduces the RMS residuals by $12 \%$ in Germany and by $47 \%$ in the U.S.. From Figs. 4 and 5, TIG often reduces the residuals between SIG and ground-truth quasigeoid undulations by $\sim 1 \mathrm{~m}$ or more in rugged terrain. Medium-scale oscillations (Fig. 4) are assumed to reflect Gibbs phenomena originating from the spherical harmonic topography used to construct the RTM data. 
Table 5 Comparisons using ground-truth quasigeoid undulations

\begin{tabular}{|l|c|l|c|c|c|c|c|}
\hline Dataset & \multirow{2}{*}{ Points } & \multicolumn{1}{|c|}{ Difference } & \multicolumn{3}{|c|}{ Descriptive Statistics [m] } & Improve \\
\cline { 3 - 7 } & & & Min & Max & Mean & RMS & ment \\
\hline $\begin{array}{l}\text { Germany } \\
\text { GPS/lev* }\end{array}$ & \multirow{2}{*}{675} & Observed-SIG & -1.5 & 1.6 & 0.0 & 0.39 & \multirow{2}{*}{$12 \%$} \\
\cline { 3 - 7 } & & Observed-(SIG+TIG) & -1.1 & 1.2 & 0.0 & 0.35 & \\
\hline $\begin{array}{l}\text { United States } \\
\text { GPS/lev }\end{array}$ & \multirow{2}{*}{1647} & Derived -SIG & -2.1 & 3.6 & 0.0 & 0.63 & \multirow{2}{*}{$47 \%$} \\
\cline { 3 - 7 } & & Derived -(SIG+TIG) & -1.4 & 1.2 & 0.0 & 0.34 & \\
\hline
\end{tabular}

* = Bias-fit applied + = Bias- and tilt-fit applied
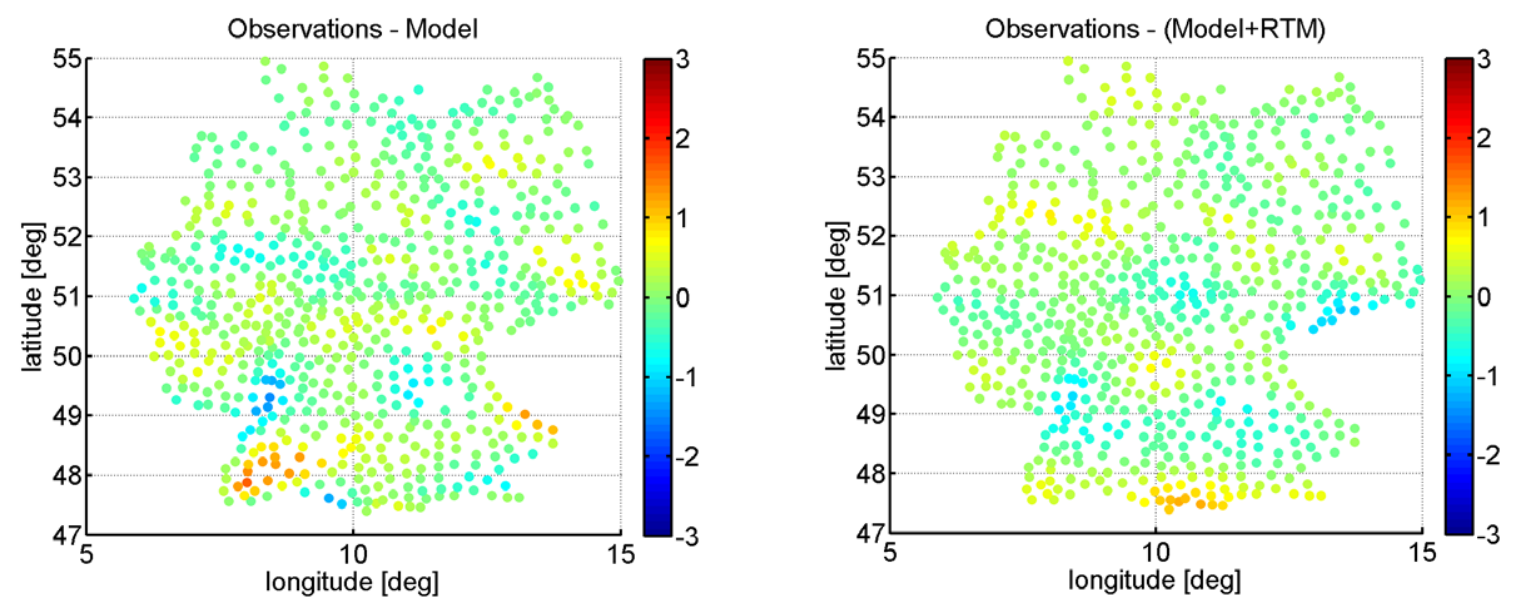

Fig. 4. Comparison of quasigeoid undulations from GPS and spirit levelling over Germany. Left: ground-truth geoid minus GOCO01S (band 2 to 160) geoid. Right: ground-truth geoid minus GOCO01S geoid minus RTM geoid. Units in metres.
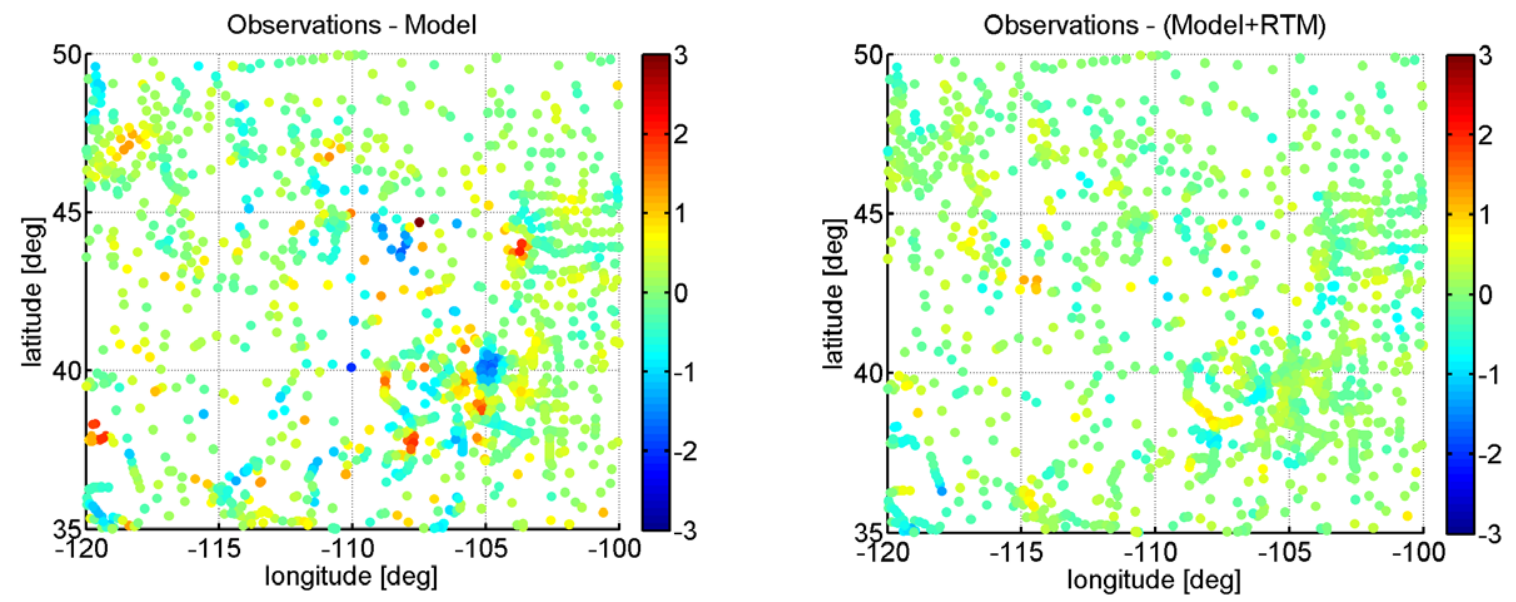

Fig. 5. Comparison of quasigeoid undulations from GPS and spirit levelling over parts of the U.S. Left: groundtruth geoid minus GOCO01S (band 2 to 160) geoid. Right: ground-truth geoid minus GOCO01S geoid minus RTM geoid. Units in metres. 


\section{Global comparisons between topography and gravity of Mars and Earth}

This section compares SIG and TIG for Earth and Mars in a comparative manner, whereby we do not use RTM-data to derive TIG, but spherical harmonic models of the planet's global topography (Appendices A1 and A2). As a function of the spatial scale (half-wavelength), we analysed and compared signal strengths, cross-correlation and reduction rates (see Appendix A3). For Mars, SIG and TIG exhibit very similar RMS signal strengths at spatial scales of $\sim 500 \mathrm{~km}$ and shorter (Fig. 6a). Opposed to this, gravity disturbances implied by the Earth's topography have much higher spectral power than SIG at all scales shown in Fig. 6b.
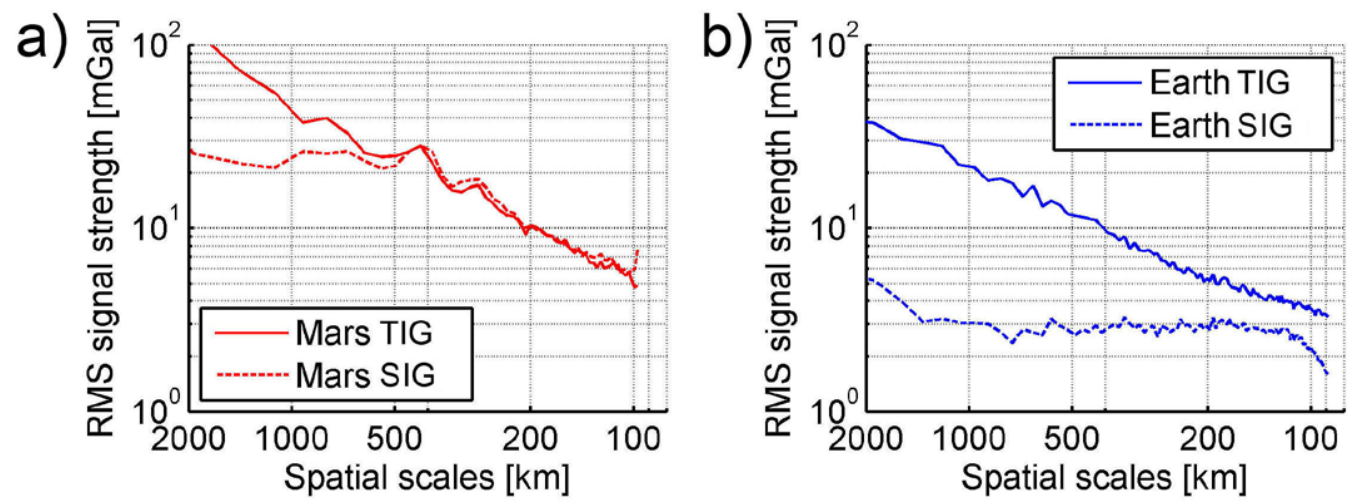

Fig. 6. Comparison between the RMS signal strengths of SIG and TIG for Earth (left) and Mars (right). Gravity models used: Earth: GOCO01S; Mars: MRO110B2. Topography models used: Earth: RET2011 developed at Curtin University based on DTM2006.0, Mars: MarsTopo719. Topography was converted to gravity using the potential coefficient transformation by Rummel et al. (1988), see Appendix A1.

Figure 6 demonstrates the effect of isostatic compensation of the topographic masses, which represents a significant effect for Earth's long- and medium-wavelength gravity field (e.g., Watts, 2001; Göttl and Rummel, 2009). From Fig. 6a, the comparable signal strengths of SIG and TIG coincide much closer for Mars at scales shorter $\sim 500 \mathrm{~km}$. This demonstrates that isostatic compensation plays a lesser role for Mars's than Earth's medium-wavelength gravity field.

Figure 7a displays the cross-correlation between SIG and TIG for Mars and for Earth. The declining correlation for Mars occurring at scales below $\sim 125 \mathrm{~km}$ reflects the effect of attenuation of MRO110B gravity. The same effect is visible for GOCO01S at scales of $\sim 100$ $\mathrm{km}$. At identical spatial scales, the cross-correlation between SIG and TIG is larger for Mars than Earth (Fig. 7a). This shows that the (uncompensated) topography is a more dominant source for Mars's than Earth’s high-frequency gravity field. Similar correlation coefficient 
curves were published by Wieczorek, (2007). By way of background, space-collected gravity is better resolved for Earth due to high-resolution GOCE satellite gravimetry data contained in GOCO01S.
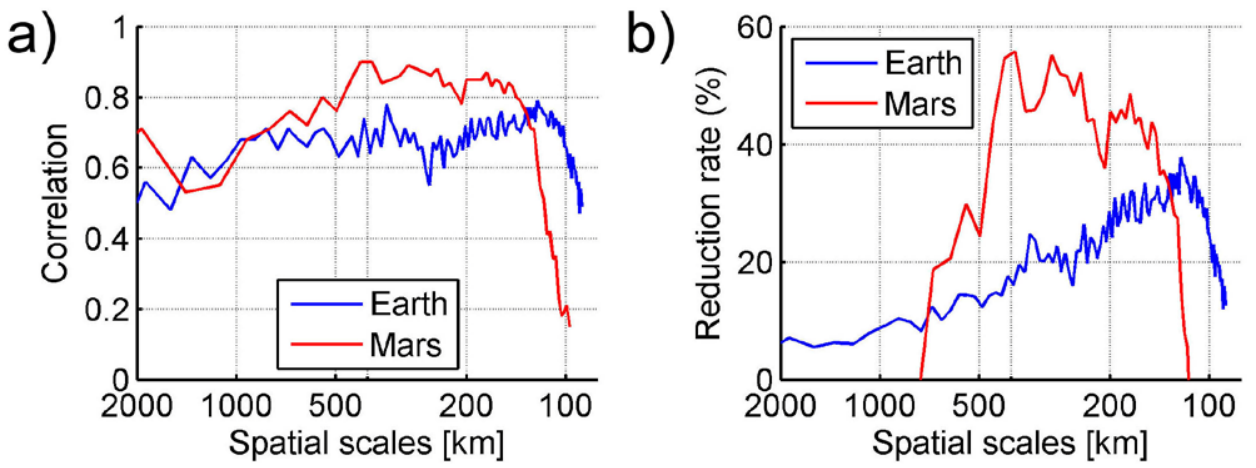

Fig. 7. Correlation between SIG and TIG for Mars and Earth as a function of the spatial scale (left) and reduction rates (quantifying the extent of topography signals captured by the space-collected gravity; Appendix A3) for Mars and Earth as a function of the spatial scale (right). The correlation was computed between SIG (Earth: GOCO01S; Mars: MRO110B2) and TIG (Earth: from RET2011; Mars: from MarsTopo719).

Another indicator to describe the relation between gravity and topography are reduction rates (Appendix A3), which quantify the proportion of TIG signals captured by the SIG. From the reduction rates in Fig. $7 \mathrm{~b}, 40-50 \%$ of Mars SIG is topography-generated at scales of a few $100 \mathrm{~km}$, while on Earth only 20-30\% of observed gravity is explained by the topography at the same scales. Again, a reasonable explanation for these differences is that isostatic compensation is more prevalent for the Earth's than Mars's gravity fields at medium scales. A more detailed discussion of isostatic compensation of Earth and Mars at different spatial scales is beyond the scope of the present paper, but is a work in progress. Reduction rates dropping from $\sim 40 \%$ to $0 \%$ (Fig. $7 \mathrm{~B}$, red line) demonstrate that there are no notable TIG-signals captured by MRO100B2 at scales less than $\sim 125 \mathrm{~km}$, which serves as a justification of our choice not to use the MRO110B2 in the high-degree spectral band of harmonic degrees 86 to 110 in the construction of MGM2011 (also see Hirt et al., 2012a).
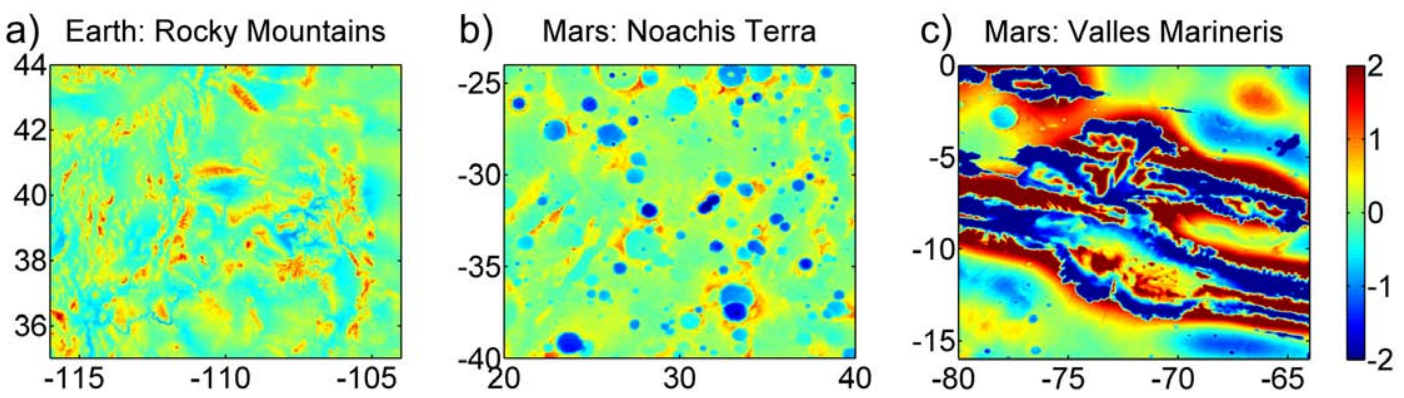
Fig. 8. Comparison of high-frequency topography of Earth (panel a) and Mars (panels b and c). Panel a shows the RTM over U.S./Canadian Rocky Mountains, Panels b and c show the RTM over parts of Mars's southern hemisphere. All areas shown are approximately $1000 \mathrm{~km} \times 1000 \mathrm{~km}$, colour scale is the same for all panels, unit is $\mathrm{km}$. RTMs constructed by removing spatial scales down to $\sim 125 \mathrm{~km}$ through subtraction of spherical harmonic topography (DTM2006 to degree 160, MarsTopo719) from the detailed elevation models (SRTM, MOLA), for Earth and Mars, respectively.

Finally, large parts of Mars's surface (with the exception of Mars's northern plains) are rugged (cf. Smith et al., 2001), often rougher than Earth's surface. This is seen from a visual comparison of Earth’s RTM topography (Rocky Mountains, Fig. 8a) with Mars’s RTM topography (Fig. 8b). On Mars, even more rugged areas exist (Vales Marineris, Fig.8c), with much stronger RTM variations than on Earth (as exemplified in Fig. 8a). As such, the Martian topography is a significant contributor to that planet's medium- and short-scale gravity field.

\section{Discussion and inferences for MGM2011}

Direct evaluation of the MGM2011 performance with ground-truth data is not possible. We have therefore created a replication of the MGM2011 modelling procedures over selected areas on Earth, so as validate the modelling technique and to indirectly test its likely performance on Mars. In replicating MGM2011 on Earth, we have paralleled the MGM2011 development as closely as possible with terrestrial data sets (Section 2). Importantly, both MGM2011 and its replication on Earth source the high-frequency signals from residual topography (RTM) and use Newtonian forward-modelling for conversion to TIG effects. For both planets, the RTM was constructed such that the spatial scales delivered are the same: $\sim 125 \mathrm{~km}$ down to $\sim 3 \mathrm{~km}$.

Over our six test areas on Earth, addition of TIG from RTM data to SIG data reduced the residuals with respect to the ground-truth data, for all test areas and for all functionals [gravity disturbances, vertical deflections and quasigeoid undulations]. The RMS improvements were substantial for gravity (55\% and 67\%) and considerable for deflections (30\% to $48 \%$ ) and quasigeoid undulations (12\% and $47 \%$ ). In a relative sense, this behaviour is within expectations, given that gravity and deflections possess significant power at short scales, while the geoid spectrum is concentrated in the long-wavelengths (cf. Schwarz 1984). 
Our Earth-based tests showed that TIG reduces the high-frequency discrepancies between SIG and ground-truth gravity, so is effective at short-scale gravity field improvement in rugged terrain. At scales shorter than $\sim 125 \mathrm{~km}$, gravity field structures were shown to exist on Earth (e.g., isostatic compensation effects over the European Alps, and the Ivrea near-surface mass-density anomaly) that are not modelled by a constant mass-density uncompensated RTM topography. Recovery of such gravity features requires observations (or more sophisticated models than the constant mass-density uncompensated RTM).

MRO110B/2 - currently the highest resolution SIG models of Mars - describe its gravity field well to degree $\sim 85$ (scales of $\sim 125 \mathrm{~km}$, as indicated from comparisons with TIG, see Fig. 7), reach a signal-to-noise ratio of 1 near harmonic degree $\sim 95$ (scales of $\sim 112 \mathrm{~km}$, cf. Konopliv et al., 2011) and provide regionally some attenuated gravity information up to harmonic degree $\sim 110$ (scales of $\sim 97$ km, see Konopliv et al., 2011). However, beyond 97 $\mathrm{km}$ spatial scales, TIG-modelling is currently the only alternative to estimate Mars's shortscale gravity field, and our MGM2011 replication experiment on Earth demonstrates that the approach can be effective for some proportion of gravity field improvement in rugged terrain.

What inferences can be made for MGM 2011 based on our Earth-laboratory comparisons (Section 4) and comparisons between Mars's and Earth's topography and gravity (Section 5)? The comparisons between TIG and SIG demonstrate that isostatically uncompensated topography is more dominant for Mars's than Earth's gravity field at spatial scales of a few $100 \mathrm{~km}$ and less. This suggests that, if ground-truth gravity data sets were available on Mars's surface, at least comparable improvements should be seen for MGM2011 over areas where Mars's actual crustal mass-density is close to our assumed value of 2900 $\mathrm{kg} / \mathrm{m}^{3}$, e.g., over large parts of Mars's southern hemisphere. Given that regional mass-density variations were not modelled by MGM2011, lower improvements have to be expected elsewhere, e.g., over Mars’s polar ice caps.

Our MGM2011 replication experiment on Earth has only implicitly validated the MGM2011 modelling technique, but has not validated the MGM2011 model itself. However, the technique validation together with the comparisons of Earth's and Mars's global gravity field characteristics (Section 5), provide holistically some indirect evidence that MGM2011 will approximate Mars's true gravity field more closely than purely spacecollected Martian gravity fields. 


\section{Acknowledgements}

We thank the Australian Research Council (ARC) for funding through discovery project grants DP0663020 and DP120102441. Ground-truth data for this study was kindly provided by following institutions and colleagues: Swiss gravity (Swiss Geodetic Commission, Swisstopo, Dr. Urs Marti), Canadian gravity (Gravity \& Geodetic Networks Section, Geodetic Survey Division, Geomatics Canada, Earth Sciences Sector, Natural Resources Canada, Marc Véronneau), U.S. Bouguer anomalies (National Geophysical Data Center), European vertical deflections (Swiss Geodetic Commission, ETH Zurich, Dr Beat Bürki, author's own observations), US vertical deflections (derived from National Geodetic Survey's product USDOV2009), German GPS/levelling heights (Bundesamt für Kartographie und Geodäsie, Uwe Schirmer) and US GPS/levelling data (National Geodetic Survey, product GPSonBM09). The 1-km SRTM V4.1 version was created by Dr. Andrew Jarvis. We gratefully acknowledge all data providers.

\section{Appendix}

\section{A1 Conversion of topography to potential}

The fully-normalised spherical harmonic coefficients $\overline{H C}_{n m}$ and $\overline{H S}_{n m}$ of the topography $H$ can be converted to gravitational potential coefficients $\bar{C}_{n m}^{T I G}$ and $\bar{S}_{n m}^{T I G}$ using the potential coefficient transformation (after Rummel et al., 1988; Kuhn and Featherstone, 2003):

$$
\left\{\begin{array}{l}
\bar{C}_{n m}^{\mathrm{TIG}} \\
\bar{S}_{n m}^{\mathrm{TIG}}
\end{array}\right\}=\frac{4 \pi}{2 n+1} \frac{a^{2}}{M} \rho\left[\left\{\begin{array}{l}
\overline{H C 1}_{n m} \\
\overline{H S 1}_{n m}
\end{array}\right\}+\frac{n+2}{2}\left\{\begin{array}{l}
\overline{H C}_{n m} \\
\overline{H S}_{n m}
\end{array}\right\}+\frac{(n+2)(n+1)}{6}\left\{\begin{array}{l}
\overline{H C}_{n m} \\
\overline{H S}_{n m}
\end{array}\right\}\right]
$$

with $n$ harmonic degree and $m$ harmonic order, $a$ equatorial radius of the planet, $M$ mass of the planet and $\rho$ mass-density of the topography, whereby the standard values of $2670 \mathrm{~kg} / \mathrm{m}^{3}$ for Earth (e.g., Torge, 2001) and $2900 \mathrm{~kg} / \mathrm{m}^{3}$ for Mars (Wieczorek and Zuber, 2004) are used. Variables $\overline{H C 1}_{n m}, \overline{H S 1}_{n m}$ are the dimensionless coefficients of the surface function $H 1=H a^{-1}, \overline{H C 2}_{n m}, \overline{H S}_{n m}$ denote the spherical harmonic coefficients of $H 2=H^{2} a^{-2}$ and $\overline{H C 3}_{n m}, \overline{H S 3}_{n m}$ are the coefficients of $H 3=H^{3} a^{-3}$. As spherical harmonic topography models, we use MarsTopo719 for Mars (Wiecorek, 2007) and RET2011 for Earth. RET2011 is a rock-equivalent topography model constructed at Curtin University based on the DTM2006.0 model (Pavlis et al., 2007). RET2011 compresses the ocean water masses and major ice shields of Greenland and Antarctica into rock-equivalent topography. 


\section{A2 Conversion of potential to gravity}

The $\bar{C}_{n m}^{\text {TIG }}, \bar{S}_{n m}^{\text {TIG }}$ coefficients of the topography's gravitational potential are converted to TIG $\delta g^{\text {TIG }}$ (specifically, gravity disturbances) using the spherical-harmonic series expansion (e.g., Torge, 2001)

$\delta g^{\mathrm{TIG}}=\frac{G M}{R^{2}} \sum_{n=n_{1}}^{n_{2}}\left(\frac{a}{R}\right)^{n}(n+1) \sum_{m=0}^{n}\left(\bar{C}_{n m}^{\mathrm{TIG}} \cos m \lambda+\bar{S}_{n m}^{\mathrm{TIG}} \sin m \lambda\right) \bar{P}_{n m}(\cos \theta)$

where $G M$ is the product of the Universal gravitational constant $G$ and the planet's total mass $M$ and $a$ is equatorial radius of the planet, and $\bar{P}_{n m}(\cos \theta)$ are the fully-normalised associated Legendre functions of degree $n$ and order $m$. The computation points are specified by geocentric radius $R$, longitude $\lambda$ and geocentric co-latitude $\theta$. By inserting the spherical harmonic coefficients $\bar{C}_{n m}^{\text {SIG }}, \bar{S}_{n m}^{\text {SIG }}$ of the SIG-models (GOCO01S for Earth, MRO110B2 for Mars), and using the model-specific constants $G M^{\text {SIG }}$ and $a^{\text {SIG }}$ instead of $G M$ and $a$, Eq. 2 is also used to compute the SIG $\delta g^{S I G}$.

\section{A3 Reduction rates}

To quantify the relation between $\delta g^{\text {TIG }}$ and $\delta g^{\text {SIG }}$, we use cross-correlation coefficients and reduction rates (RR), the latter of which are computed by

$$
R R=100 \% \cdot \frac{R M S\left(\delta g^{\mathrm{TIG}}\right)-R M S\left(\delta g^{\mathrm{TIG}}-\delta g^{\mathrm{SIG}}\right)}{R M S\left(\delta g^{\mathrm{TIG}}\right)}
$$

where RMS is the root mean square of the $\delta g^{\text {TIG }}$ and the differences $\left(\delta g^{\text {TIG }}-\delta g^{\mathrm{SIG}}\right)$, respectively (Hirt et al., 2012b). The RMS operator gives the signal strengths of $\delta g^{\text {TIG }}$ and the differences $\left(\delta g^{\text {TIG }}-\delta g^{\text {SIG }}\right)$. Reduction rates quantify the proportion of $\delta g^{\text {TIG }}$ captured by the SIG at various spatial scales. Large positive RRs, say 50-60\%, indicate significant TIG signals are captured by the SIG, whereas RRs near or below 0\% show that the SIG signal is unrelated to the topography, , see also Hirt et al., (2012b).

\section{References}

Ardalan A.A., Karimi R. and Grafarend E.W., 2009. A new reference equipotential surface, and reference ellipsoid for the planet Mars. Earth Moon Planet, 106, 1-13. 
Bürki B., 1989. Integrale Schwerefeldbestimmung in der Ivrea-Zone und deren geophysikalische Interpretation. Geodätisch-geophysikalische Arbeiten in der Schweiz, Nr. 40, Schweizerische Geodätische Kommission. Featherstone, W.E., 1995. On the use of Australian geodetic datums in gravity field determination, Geomatics Research Australasia 62: 17-36.

Forsberg R., 1984. A study of terrain reductions, density anomalies and geophysical inversion methods in gravity field modelling. Report, 355, Department of Geodetic Science and Surveying, Ohio State University, Columbus

Göttl F. and Rummel R., 2009. A Geodetic View on Isostatic Models. Pure Appl. Geoph., 166, 1247-1260, DOI: $10.1007 / \mathrm{s} 00024-004-0489-\mathrm{x}$.

Hirt C., 2010. Prediction of vertical deflections from high-degree spherical harmonic synthesis and residual terrain model data. J. Geodesy 84, 179-190. DOI 10.1007/s00190-009-0354-X.

Hirt C., Featherstone W.E., and Marti U., 2010a. Combining EGM2008 and SRTM/DTM2006.0 residual terrain model data to improve quasigeoid computations in mountainous areas devoid of gravity data. J. Geodesy, 84(9. 557-567, DOI 10.1007/s00190-010-0395-1.

Hirt C., Marti U., Bürki B. and Featherstone W.E., 2010b. Assessment of EGM2008 in Europe using accurate astrogeodetic vertical deflections and omission error estimates from SRTM/DTM2006.0 residual terrain model data. J. Geophys. Res. Solid Earth, 115, B10404, DOI:10.1029/2009JB007057.

Hirt C., Claessens S.J., Kuhn M. and Featherstone W.E., 2012a. Kilometer-resolution gravity field of Mars: MGM2011. Planetary and Space Science, paper in press, DOI: 0.1016/j.pss.2012.02.006

Hirt C., Kuhn M., Featherstone W.E., and Göttl F., 2012b. Topographic/isostatic evaluation of new-generation GOCE gravity field models, J. Geophys. Res. Solid Earth, paper in press, DOI:10.1029/2011JB008878.

Holmes, S.A. and Pavlis N.K., 2008. Spherical harmonic synthesis software harmonic_synth. (http://earth-info.nga.mil/GandG/wgs84/gravitymod/new_egm/new_egm.html)

Ihde J. and Sacher M., 2002. EUREF Publikation 11/I. Mitteilungen des Bundesamtes für Kartographie und Geodäsie, Band 25, Frankfurt/Main, ISBN 3-89888-869-X.

Jarvis A., Reuter H.I., Nelson A., and Guevara E., 2008. Hole-filled SRTM for the globe Version 4. Available from the CGIAR-SXI SRTM 90m database (http://srtm.csi.cgiar.org).

Jekeli C., 1999, An analysis of vertical deflections derived from high-degree spherical harmonic models, J Geod 73, 10-22, doi: 10.1007/s001900050213

Konopliv A.S., Asmar S.W., Folkner W.M., Karatekin Ö., Nunes D.C., Smrekar S.E., Yoder C.F. and Zuber M.T., 2011. Mars high resolution gravity fields from MRO, Mars seasonal gravity, and other dynamical parameters. Icarus, 211, 401-428.

Kuhn, M. and Featherstone W.E., 2003. On the optimal spatial resolution of crustal mass distributions for Forward Gravity Field Modelling. In: Proceed 3rd Meeting of the Intern. Gravity and Geoid Commission, (ed. I Tziavos), Editions Ziti, pp 195-200.

Marti U., 2004. High-precision combined geoid determination in Switzerland. Presented at Gravity, Geoid and Space Missions (GGSM) 2004 Symposium, Porto, Aug 30 - Sep 03 2004, Porto, Portugal

Moritz H., 1980. Geodetic Reference System 1980. J. Geodesy, 54, 395-405.

Moritz H., 2000. Geodetic Reference System 1980. J. Geodesy, 74, 128-133. 
Nagy D., Papp G., and Benedek, J., 2000. The gravitational potential and its derivatives for the prism $J$. Geodesy, 74, 552-560, Erratum in J. Geodesy, 76, 475.

NGDC 2011. 2.5-min gravity grid of N. America. National Geophysical Data Center, US Department of Commerce and National Oceanic Atmospheric Administration, USA. (http://www.ngdc.noaa.gov/ngdc.html)

NGS 2011a. U.S Vertical Deflections 2009. National Geodetic Survey, USA, ( http://www.ngs.noaa.gov/GEOID/USDOV2009/).

NGS 2011b. GPS on Benchmark Data 2009. National Geodetic Survey, USA, ( http://www.ngs.noaa.gov/GEOID/GPSonBM09/).

NRC 2011. Canadian gravity data base. Natural Resources Canada, Canada, ( http://gdcinfo.agg.nrcan.gc.ca/cat/indexgrav_e.html).

Pail R., Goiginger H., Schuh W.-D., Höck E., Brockmann J.M., Fecher T., Gruber T., Mayer-Gürr T., Kusche J., Jäggi A., and Rieser D., 2010. Combined satellite gravity field model GOCO01S derived from GOCE and GRACE. Geophys. Res. Lett. 37, L20314, DOI: 10.1029/2010GL044906.

Pavlis N.K., Factor J.K. and Holmes S.A., 2007. Terrain-related gravimetric quantities computed for the next EGM. Proceedings of the 1st International Symposium of the International Gravity Field Service (IGFS), Istanbul, pp 318-323.

Rapp R.H., 1997. Use of potential coefficient models for geoid undulation determinations using a spherical harmonic representation of the height anomaly/geoid undulation difference. J. Geodesy, 71, 282-289.

Rummel R., Rapp R.H., Sünkel H., and Tscherning C.C., 1988. Comparisons of global topographic/isostatic models to the Earth's observed gravity field. Report 388, Dep. Geodetic Sci. Surv., Ohio State University, Columbus, Ohio.

Rummel R., Yi W., and Stummer C., 2011. GOCE gravitational gradiometry. J. Geodesy, 85, 777-790, DOI 10.1007/s00190-011-0500-0.

Schwarz, K-P., 1984. Data types and their spectral properties, in: Schwarz (ed) Local gravity field approximation, Beijing International Summer School.

Smith D.A., 1998. There is no such thing as "The” EGM96 geoid: Subtle points on the use of a global geopotential model, International Geoid Service Bulletin 8, 17-28.

Smith D.E., Zuber M.T., Frey H.V., Garvin J.B., Head, J.W. et al., 2001. Mars Orbiter Laser Altimeter (MOLA): experiment summary after the first year of global mapping of Mars. J. Geophys. Res. 106 (E10), 23689-23722.

Tapley B.D., Bettadpur S., Watkins M. and Reigber C., 2004. The gravity recovery and climate experiment: Mission overview and early results. Geophys. Res. Lett., 31, L09607, doi:10.1029/2004GL019920.

Torge W., 2001. Geodesy, 3rd Edition, de Gruyter, Berlin, New York.

Wang Y.M., Saleh, J., Li, X., and Roman, D.D., 2011. The US Gravimetric Geoid of 2009 (USGG2009): model development and evaluation. J Geodesy, online first, DOI: 10.1007/s00190-011-0506-7.

Watts A.B., 2001. Isostasy and Flexure of the Lithosphere. Cambridge University Press, 458 pp.

Wieczorek M.A., 2007. Gravity and topography of the terrestrial planets. In: Treatise on Geophysics, 10, 165206. Elsevier-Pergamon, Oxford.

Wieczorek M.A., and Zuber M.T., 2004. Thickness of the Martian crust: Improved constraints from geoid-totopography ratios. J. Geophys. Res., 109, E01009. 